\title{
SUMER/SOHO Filament Observations in Selected Lyman Lines
}

\author{
B. Schmieder ${ }^{1}$ \\ Observatoire de Paris, Section Meudon, F 92195 Meudon, France
}

T. Kucera ${ }^{2}$

NASA, GSFC, MD 20771, USA

P. Heinzel

Astronomical Institute, CZ 25165, Ondřejov, Czech Republik

J.-C. Vial

Institut d'Astrophysique Spatiale, CNRS/Université Paris XI, Bât. 121, 91405 Orsay Cedex, France

\begin{abstract}
.
On September 21, 1996 a filament located in a region close to an enhanced network was observed with the SOHO SUMER and CDS instruments. Four Lyman lines have been detected (L $\delta, \mathrm{L} \epsilon, \mathrm{L}-6, \mathrm{~L}-7$ ) by SUMER in the raster mode. We have corrected the spectral data for flatfield and destreching and made wavelength and absolute intensity calibrations. In all these lines we detect a central absorption and an asymmetry in the intensity of the two peaks. Preliminary NLTE computations indicate that these Lyman profiles and their absolute intensities can be reproduced with the existing models provided that we take into account a prominence-corona transition region (PCTR).
\end{abstract}

\section{Introduction}

The diagnostic potential for quiescent prominences of Lyman lines has been evidenced in the works of Gouttebroze et al. (1993) and Heinzel et al. (1994). These authors have shown, for instance, how the Lyman alpha and the Lyman beta lines (differently radiatively dominated) could be indicators of temperature and pressure (at higher values).

In the case of (radially) moving prominences, Gontikakis et al. (1997a, b) have shown evidence that the Lyman beta profile is still sensitive to Doppler effects, even if less so than Lyman alpha.

\footnotetext{
${ }^{1}$ Institute of Theoretical Astrophysics, P.O. Box 1029, Blindern, N-0315 Oslo, Norway

${ }^{2}$ Applied Research Corporation, 8201 Corp. Drive, Landover, MD 20785, USA
} 


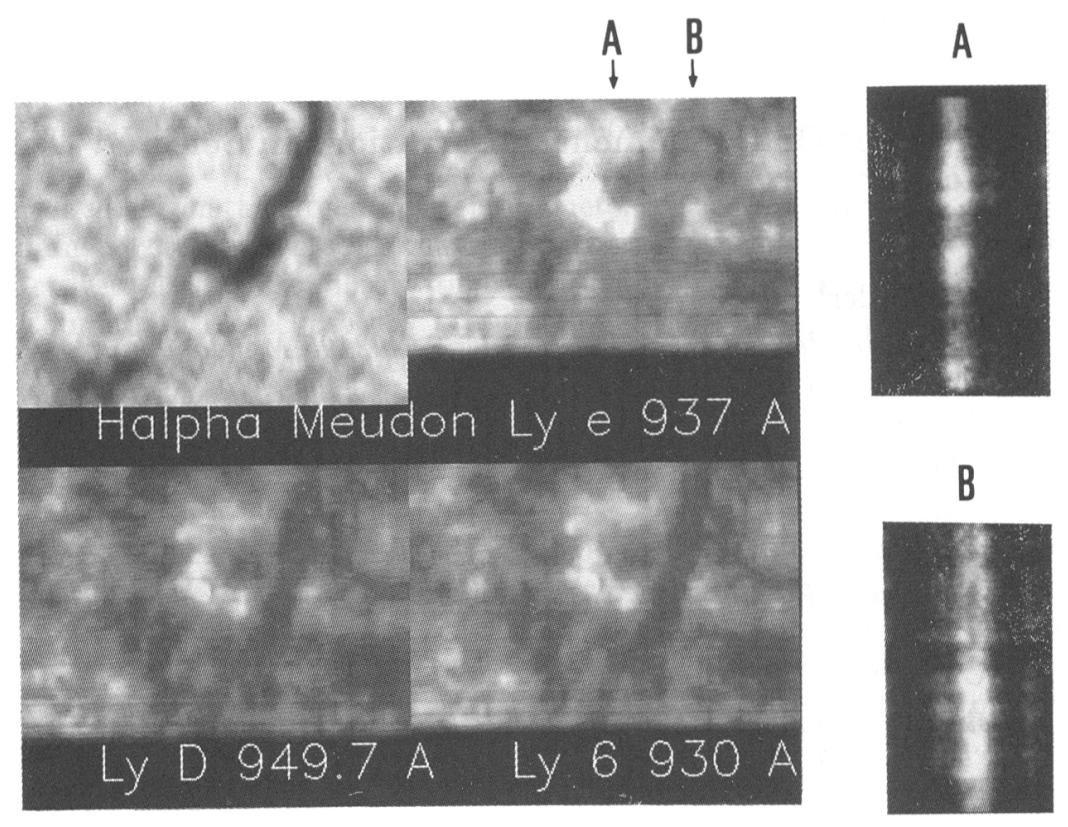

Figure 1. Left panels (a): Observations of the filament on September 21, 1996: at Meudon in H $\alpha$ and with SUMER on SOHO in Lyman lines (only 100 pixels along the slit are used in these images). The Lyman line intensities are averaged over 3 pixels at the profile center. The arrows indicate the positions $\mathrm{A}$ and $\mathrm{B}$ of the spectra shown in the right panels (b): examples of Lyman spectra observed by SUMER in A through the network and in $B$ in the filament.

Going higher in the Lyman series, we expect that the shapes and intensities of these optically thick lines can provide information on the temperature, radial velocity and density across the observed structure. We stress that this information is not limited to low temperatures only.

\section{SUMER Observations and Data Reduction}

The target of SUMER on board the SOHO satellite was part of a North-South filament (S6-12, E5), close to a bright network cell (see $\mathrm{H} \alpha$ spectroheliogram from Meudon, Figure 1a). SUMER has been described by Wilhelm et al. (1997). SUMER was rastering a region 120 by 150 arc sec with a raster-step of 3 arc sec. The exposure time was $60 \mathrm{sec}$ with the slit-width being 1 arc sec. In the wavelength range 920 to $950 \AA$, seven windows of $50 \times 120$ pixels each were displayed on the detector A, with one window (949.7 $\AA$ ) being on the bare part of the detector. Seven lines have been observed: S VI at 944.54 and $933.380 \AA$, N IV at $923.220 \AA$ and four lines of the Lyman series: L $\delta$ (949.74), L $\epsilon$ (937.80), L-6 (930.75) and L-7 (926.23 $\AA$ ).

We first corrected the observations by a flat-field image obtained on Septem- 

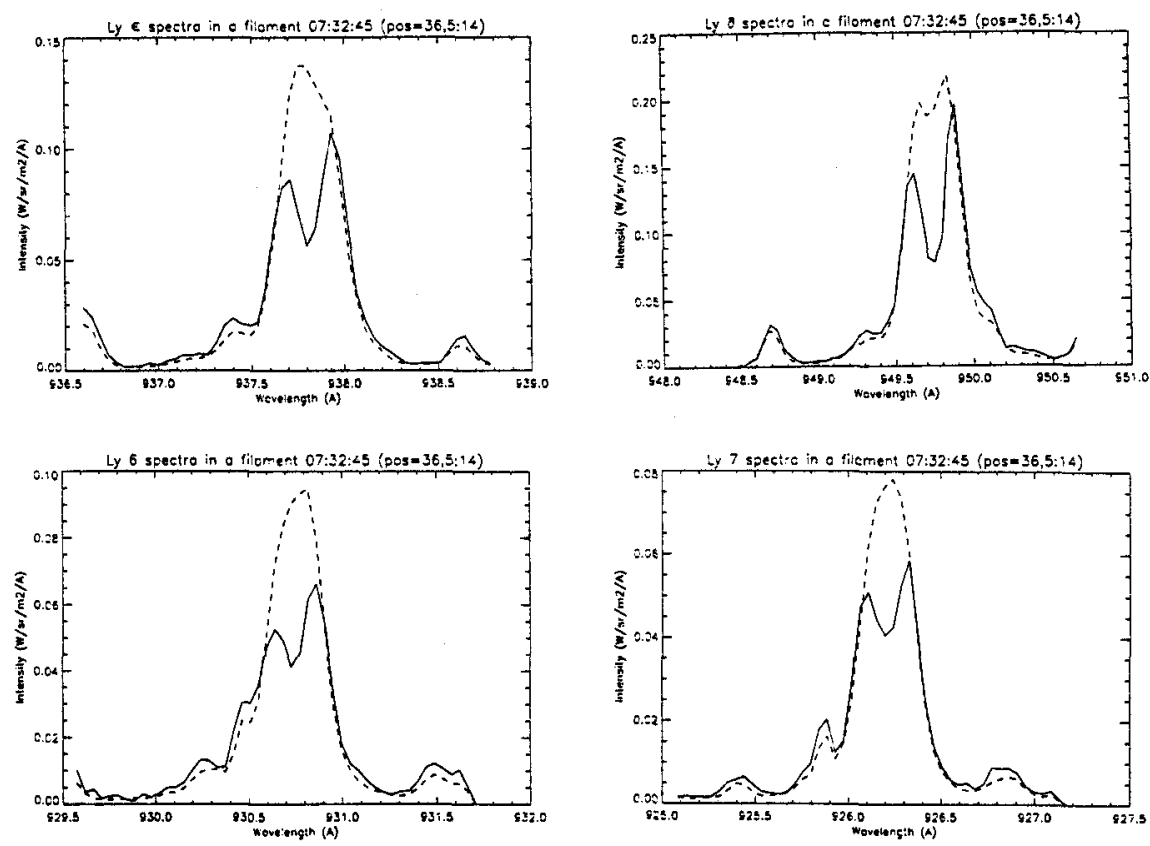

Figure 2. Examples of profiles in the Lyman series. The dashed profiles correspond to the background emission, obtained by suming 5 spectra. The solid ones correspond to the filament (average profile over 10 pixels along the slit).

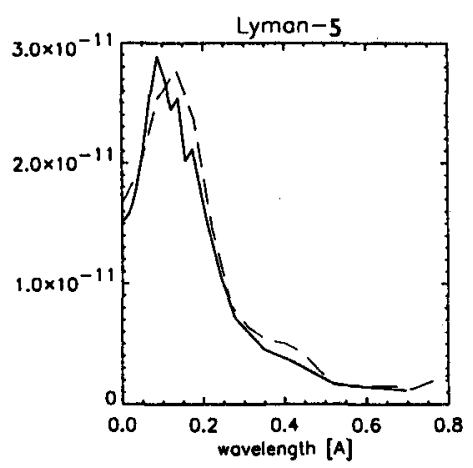

Figure 3. Fit of the observed $L \epsilon$ profile (dashed line) with the theoretical one (solid line). 
ber 24, 1996. The curvature of the lines ('inverse-cushion' distortion) depends on their position on the detector. The 'destretching' of the spectra was done with the SUMER analysis software procedure destrech.pro provided by T. Moran.

Wavelength calibration was done by using the Lyman lines observed on the quiet Sun nearby. The profiles were fitted by gaussians. We derived the mean dispersion: $0.04445 \AA$ /pixel. We found that the measured wavelengths are shifted relative to the standard wavelengths by quantities less than $\mathbf{0 . 0 1 5}$ pixel, a value which corresponds to one third of a pixel $(4 \mathrm{~km} / \mathrm{s})$.

For the intensity calibration we used the procedure of Wilhelm et al. (1997) called radiometry-a. Our results are consistent with the spectra obtained by Wilhelm for the quiet Sun but our values of the line maxima are higher by a factor 1.3 , a discrepancy that we explain by the presence of a network boundary in the field (Figure 1a, b).

\section{Comparison of Observed and Theoretical Profiles}

In Figure 2 we give examples of the four Lyman lines that were observed in the filament. All filament profiles of Lyman lines have central reversals because of self absorption. They also are asymmetrical (Schmieder et al. 1998). NLTE computations (see Heinzel 1998, these proceedings) show that an isothermal model cannot reproduce profiles of all lines. Using a 1D horizontal slab model and the NLTE tranfer code based on the MALI-method, we show that a reasonable fit for L $\epsilon$ can be obtained with a PCTR model $\left(T_{c}=7000 \mathrm{~K}\right.$ and $T_{s}=14000$ $\mathrm{K}, D=20000 \mathrm{~km}, p=0.1 \mathrm{dyn} \mathrm{cm}^{-2}, \mathrm{v}_{t}=10 \mathrm{~km} \mathrm{~s}^{-1}$ ) (Figure 3). However, to reproduce all lines simultaneously one has to apply an optimization technique to construct the filament model.

\section{References}

Gouttebroze, P., Heinzel, P. and Vial, J.-C. 1993, A\&AS, 99, 513

Heinzel P., Gouttebroze P. and Vial J.-C. 1994, A\&A, 292, 656

Gontikakis, C., Vial, J.-C. and Gouttebroze, P. 1997a, Solar Phys., 172, 189

Gontikakis, C., Vial, J.-C. and Gouttebroze, P. 1997b, A\&A, 325, 803

Schmieder, B., Heinzel, P., Kucera, T. and Vial, J.-C. 1998, Solar Phys., in press Wilhelm K. et al. 1997, Solar Phys. 170, 75 